\title{
Saponins extracted from Polemonium caeruleum have adjuvant activity in guinea pig intranasal immunization with trivalent influenza antigens
}

\author{
Evseenko VA ${ }^{1 *}$, Gudymo AS ${ }^{1}$, Danilchenko NV ${ }^{1}$, Onkhonova GS ${ }^{1}, \mathrm{Vu} \mathrm{LT}^{2}$ and Ryzhikov AB ${ }^{1}$ \\ ${ }^{1}$ State Research Center of Virology and Biotechnology Vector (SRC VB “Vector”) Rospotrebnadzor, Koltsovo, Novosibirsk Region, 630559, Russian Federation \\ ${ }^{2}$ Vietnamese-Russian Tropical center, Head office, Institute of Tropical medicine, Hanoi, Vietnam
}

\begin{abstract}
Saponins extracted from Polemonium caeruleum were studied as intranasal adjuvant by guinea pig intranasal immunization with trivalent influenza antigens. Sera titers of the animals immunized intranasally and control animals immunized intramuscularly were measured in HI and ELISA. On day 21 reciprocal GMT HI titers in group dually immunized intranasally were 201/64/31 against H1N1pdm09/H3N2/B components accordingly. Data obtained in ELISA shows that there was no statistically significant $(\mathrm{P}=0.95)$ differences in sera titers with control group of single intramuscularly immunized animals. Presented research shows that Polemonium caeruleum saponins can be studied as a candidate mucosal adjuvant for intranasal or oral viral or bacterial inactivated vaccines.
\end{abstract}

\section{Introduction}

Lower respiratory tract infections and pneumonia are two of the leading causes of death, accounting for more than 4 million fatalities annually. It is a particularly important cause of death in low- and middle-income countries. Streptococcus pneumonia remains the most frequent bacterial cause of pneumonia and killed 393,000 children aged under 5 years in 2015 [1]. Respiratory syncytial virus (RSV) is the most common cause of acute respiratory infection in children, causing almost 34 million episodes annually. In 2003, severe acute respiratory syndrome (SARS), caused by a previously unrecognized coronavirus, rapidly spread throughout the world. Vaccines against RSV, SARS are still under development. Vaccines against Influenza are tri- or quadrivalent, inactivated with or without adjuvant or live-attenuated but both live and inactivated ones are criticized for their low efficacy $[2,3]$.

Intranasal inactivated vaccines have a number of advantages: cause both humoral, local cellular and mucosal response, do not require qualified medical staff for the injection and safe in terms of life virus issues. Promising inactivated intranasal trivalent influenza virus vaccine for active immunization against influenza by the nasal route had been developed. It consists of influenza virosomes which are formulated from inactivated influenza strains and heat-labile toxin from aseptic Escherichia coli bacteria strain, as an adjuvant. This vaccine which had been licensed and distributed in Switzerland in 2000-2002 but later was shown to be associated with Bell's palsy and recalled from the market [4-6]. Advantages of intranasal and sublingual delivery of inactivated polio vaccine were emphasized in experiments with Cholera toxin from Vibrio Cholerae as a mucosal adjuvant [7]. Due to the safety reasons modified and non-modified bacterial toxins and components as well as chemical substances without long history of intranasal administration have serious medical risks. However, mannatide, which is also known as alpha-polyactin or polyactin A is a heteropolysaccharide isolated from the fermentation broth of buccal alpha-hemolytic Streptococcus strain was shown as promising mucosal adjuvant [8].

Another perspective class of adjuvants is immunogenic saponins. Nanoparticles from plant saponins as delivery system for mucosal influenza vaccine was tested as mucosal adjuvant as a part of ISCOMs [9]. Saponin-based adjuvants (SBAs) induce cross-presentation in dendritic cells. SBAs induce potent immune responses to a broad repertoire of antigens. The induction of potent CD8+ T-cell responses is dependent on the ability of SBAs to facilitate cross-presentation of exogenous antigens in MHC-I by DCs. Dendritic cells have a critical role in the in vivo effectiveness of SBA-aided vaccines, and enhanced antigen cross-presentation by dendritic cells has been reported to be particularly important for the ability of SBAs to induce cellular CD8+ T-cell immunity [10]. Saponins are also well known as substance with hemolytic activity. Saponins integrate with their hydrophobic part (sapogenin) into the cell membrane. Within the membrane they form complexes with sterols, which subsequently, driven by interaction of their extra-membranous orientated saccharide residues, accumulate into plaques. Sterical interference of these saccharide moieties causes membrane curvature subsequently leading to pore formation in the membrane or hemitubular protuberances resulting in sterol extraction via vesiculation [11]. This process could be biologically estimated and standardized by hemolytic reaction in microplates. In contrast with

${ }^{*}$ Correspondence to: Vasily Aleksandrovich Evseenko, State Research Centre of Virology and Biotechnology Vector (SRC VB "Vector") Rospotrebnadzor, Koltsovo, Novosibirsk Region, 630559, Russian Federation, E-mail: evseenko_va@vector.nsc.ru; vasily.evseenko@gmail.com

Key words: vaccine, intranasal, adjuvant, saponin, polemonium, influenza

Received: January 09, 2020; Accepted: January 22, 2020; Published: January 28, 2020 
bacterial toxins, some saponins as a part of plant extracts are safe and used for decades as medical drugs [12]. Earlier we have shown that Cyclamen europaeum tubers extract predominantly consisted of saponins with concentration commonly safely used for human rhinosinusitis treatment tested as mucosal adjuvant in experimental intranasal immunization of guinea pigs caused reciprocal GMT titers 40 (5-640) against H1N1pdm component, 43.20 (5-1280) against H3N2 component and 10.80 (5-80) against influenza B component [13].

Polemonium caeruleum is a Chinese medicinal plant used in the treatment of tuberculosis, whooping cough, fever, infertility, epilepsy, endometritis and insomnia. It was also used as sedative and anesthetic agent [14]. In this research study we evaluate saponins extracted from Polemonium caeruleum as potential intranasal adjuvant for guinea pig immunization with trivalent influenza antigens to confirm saponins adjuvant features for intranasal administration and its vaccine potential. Polemonium caeruleum as well as Cyclamen europaeum has long safe pharmaceutical history, was used for ulcer, tuberculosis treatment and expectorant in pre-chemical era [15].

\section{Materials and methods}

Before the experiment commercially available human inactivated split trivalent influenza vaccine containing egg-derived A/ California/07/2009 H1N1pdm-like, A/Switzerland/9715293/2013 H3N2-like and B/Phuket/3073/2013-like antigens was concentrated to $300 \mu \mathrm{g}$ of each hemagglutinin per milliliter using centrifuge membrane $100 \mathrm{kDa}$ (Millipore, US) tubes according to the manufacturer manual. Hemagglutinin concentration was confirmed by dilution and following single radial immune diffusion using standards obtained from NIBSC, UK. Concentrated trivalent influenza antigen $300 \mu \mathrm{g}$ of each hemagglutinin/ml (TIA) was stored at $+4^{\circ} \mathrm{C}$ upon use.

The micronized plant material (underground parts Polemonium caeruleum) was placed in stirred tank reactor with $40 \%$ ethanol, $60 \%$ deionized water in 1:2 w/w ratios for 4 hours on $50^{\circ} \mathrm{C}$. After that extract was separated on manual press and clarified by centrifugation on 4000 $\mathrm{rev} / \mathrm{min}$ (Sigma centrifuge, $12150 \mathrm{H}$ rotor). Extract was concentrated 20 - $\mathrm{X}$ by evaporation on $80^{\circ} \mathrm{C}$ and clarified by centrifugation on $10000 \mathrm{rev} / \mathrm{min}$ (Sigma centrifuge, $12150 \mathrm{H}$ rotor). Extra purification was performed by liquid chromatography in Sephadex G-25 resin in deionized water. Fractions were collected $(430 \mathrm{~nm})$ and those with hemolytic activities pulled, filtered through $0,22 \mu \mathrm{m}$ membrane (Minisart NML, Sartorius) and lyophilized.

Before immunization purified extract was rehydrated gradually with deionized water in minimum amount for dissolution. Biological activity and dose was standardized in hemolytic reaction. Reaction was performed in polystyrol V-shape microplates (Greiner Bio One) as described earlier (13). Dilution with conversion titer 1/64 (hemolytic) - 1/128 (non hemolytic) per $50 \mu \mathrm{l}$ was taken for immunization experiment. The titer of Polemonium caeruleum tubers purified extract (PTE) was stable for more than 60 days at $+4^{\circ} \mathrm{C}$ and suitable for second immunization. TIA and PTE (1/64-1/128 hemolytic units) was mixed into compound in rate 1:1 immediately before immunization. Guinea pigs (SRC VB "Vector") 180-220 grams were placed in individual cages and quarantined for 7 days before study. Three groups of 5 animals were nominated: Group 1 for intramuscular (i.m.) antigen positive control, Group 2 for intranasal (i.n.) experimental vaccination and Group 3 i.n. negative control (i.n. antigen only). Animals from Group 3 were injected intranasally with $50 \mu \mathrm{l}$ PTE (1/32-1/64 hemolytic units) on day 1 before the study to confirm guinea pig tolerance to selected concentration and dose of PTE. On day 1 all animals from Group 1, 2 and 3 were immunized. Group 1 was immunized in hip muscle with $7.5 \mu \mathrm{g}$ of each $\mathrm{HA}$ in $50 \mu \mathrm{l}$. Intranasal immunization was performed by standard $1 \mathrm{ml}$ syringe with human size nozzle sprayer; $50 \mu \mathrm{l}$ of the compound consisting of $7.5 \mu \mathrm{g}$ of each HA and PTE (1/32-1/64 hemolytic units). On day 14 after first immunization animals from Group 2 were intranasally immunized with the same compound for the second time. Blood was collected on day 2 before immunization and on day 14, 21, 28 and 55 after immunization. Sera were treated with RDE (Denka Seiken, Japan) and studied in HI using 0.5\% v/v chicken red blood cells. Sera were stored in $-20^{\circ} \mathrm{C}$ upon analysis. HI test was performed as previously described [16]. Starting dilution of the sera was $1 / 10$. Control sera and antigens were kindly provided by CDC, US through IRR. Teardrop test was used for visual confirmation of specific hemagglutination. Reciprocal titers $<10$ counted for GMP calculation as 5 .

Briefly, ELISA was performed with Protein G conjugated with horseradish peroxidase (MP Biomedicals). Complimentary antigens (IRR, USA) were diluted 1:25 with deionized water and $50 \mu \mathrm{l}$ was dispensed into each well of polystyrol plate (SPL TestiKs Maxisorb) for complete drying. Plate was washed tree times with PBS pH 7.4 with $0.05 \% \mathrm{v} / \mathrm{v}$ Tween 20 (Sigma). Sera samples with starting dilution 1:100 in PBS pH 7.4 were titrated in U-shape serological plate and transferred to ELISA plate for incubation for 1 hour on room temperature. After triple washing with PBS pH 7.4 with $0.05 \%$ v/v Tween 20 (Sigma), Protein G 1:5000 dilution in PBS pH 7.4 with 1\% w/v BSA (Sigma) was added to each well and incubated for 1 hour on room temperature. After triple washing with TMB (3,3',5,5'-Tetramethylbenzidine, Sigma) was added. After 30 minute incubation in dark container $5 \%$ sulfuric acid was added as stop reagent. Optical density on $450 \mathrm{~nm}$ was measured with Multiscan EX (Labsystems).

Sera titer was measured as maximum dilution with $>2 \mathrm{X}$ optical density of control negative sera.

For statistical calculations reciprocal titers were used. Analysis was performed with Excel (Microsoft) as described previously [13].

\section{Results}

Data obtained in $\mathrm{HI}$ is presented in Figure 1 Sera of all animals taken before immunization tested in HI against H1N1pdm09, H3N2 and B antigens had reciprocal titers $<10$. On 14 day a.i. animals in Group 1 (antigen positive control) had GMT reciprocal HI titers 100/80/80 against H1N1pdm09/H3N2/B antigens respectively. From day 14 to 55 a.i. HI titers of animals in this group remained statistically indistinguishable $(\mathrm{P}=0.95)$ to $\mathrm{H} 3 \mathrm{~N} 2$ and $\mathrm{B}$ component. H1N1pdm09 HI titer on day 21 is statistically higher $(\mathrm{P}=0.95)$ than on 14 and 28 but indistinguishable with titer on day 55. Thus, inactivated split trivalent influenza antigen saved high immunogenicity after concentration to $300 \mu \mathrm{g} / \mathrm{ml}$ and could be antigenically identified and measured in HI. All animals of Group 2 (intranasally immunized) did not show any behavioral signs of negative impact of immunization compound. After injection animals when placed back tried to sneeze out the compound within 3-5 seconds. We consider this as normal reflex reaction. Sera reciprocal GMT HI titers on day 14 (after 1st immunization) were 10/6.3/20 to H1N1pdm09/ $\mathrm{H} 3 \mathrm{~N} 2 / \mathrm{B}$ antigens respectively. Only one animal was surprisingly highly reactive against $\mathrm{B}$ component (80). After dual immunization on day 21 (Figure 1B) we observed statistically significant $(\mathrm{P}=0.95)$ reciprocal GMP titer increasing to $\mathrm{H} 1 \mathrm{~N} 1 \mathrm{pdm} 09$ and $\mathrm{H} 3 \mathrm{~N} 2$ antigens and it were 201.1 and 63.5 respectively. For B antigen reciprocal GMP titer was 31.8. It is remarkable that for all three components there was no statistically significant $(\mathrm{P}=0.95)$ differences with intramuscularly 
A
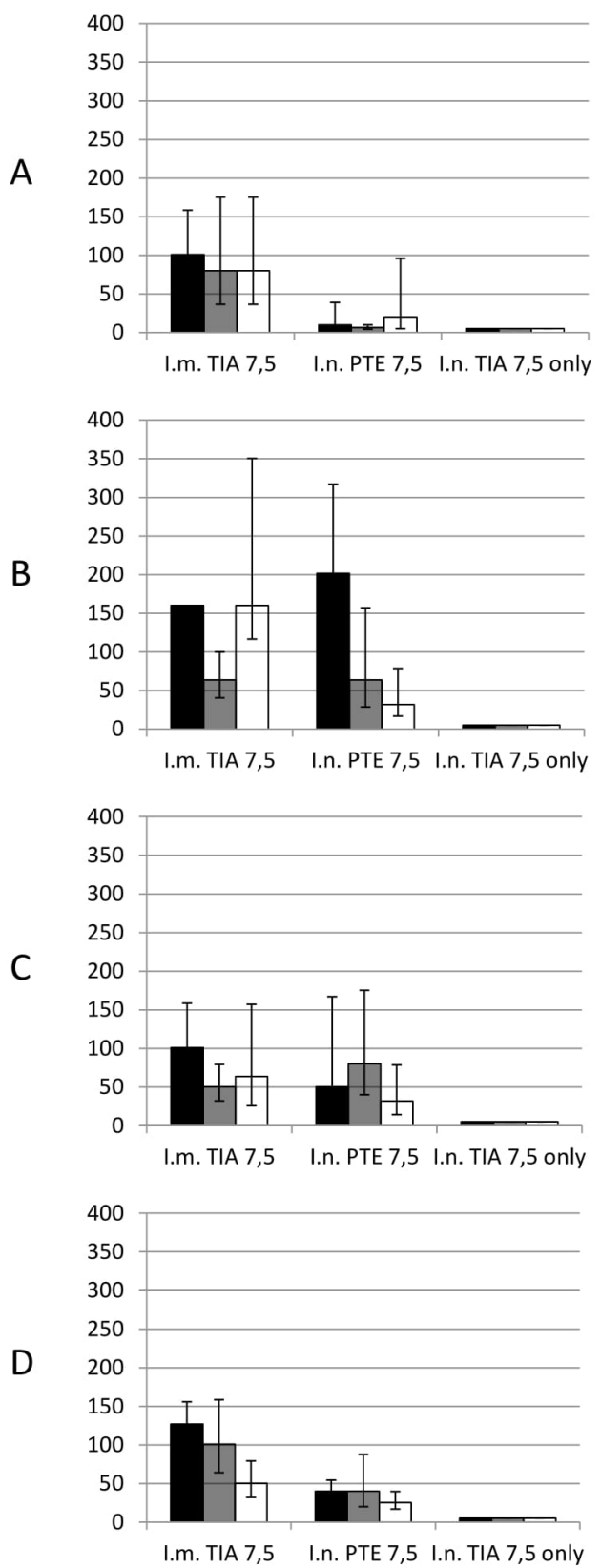

A/California/7/2009 (H1N1)pdm09-like 口 A/Switzerland/9715293/2013 (H3N2)-like 口B/Phuket/3073/2013-like

- A/California/7/2009 (H1N1)pdm09-like $\square$ A/Switzerland/9715293/2013 (H3N2)-like 口B/Phuket/3073/2013-like

\section{- A/California/7/2009 (H1N1)pdm09-like \\ $\square$ A/Switzerland/9715293/2013 (H3N2)-like \\ 口B/Phuket/3073/2013-like}

Figure 1. Anti-hemagglutinin titers in guinea pig sera measured in HA.

Reaction was performed with egg-derived antigens, $0.5 \%$ chicken erythrocytes. Data expressed as reciprocal titers (C.I. P=0.95). I.m. TIA 7.5 - intramuscular triple influenza antigen $7.5 \mu$ g each (positive antigen control). I.n. PTE + TIA 7.5 - intranasal Polemonium caeruleum tuber extract + triple influenza antigen $7.5 \mu \mathrm{g}$ each (experimental group). I.n. TIA 7.5 only - intranasal triple influenza antigen $7.5 \mu \mathrm{g}$ each (negative antigen control). A, B, C, D - 14, 21, 28, 55 days after immunization

administrated $7.5 \mu \mathrm{g}$ of each antigen and for H1N1pdm09 and H3N2 reciprocal titers were $\geq 40$ considered to be protective. Titers in sera taken on day 28 remained statistically indistinguishable $(\mathrm{P}=0.95)$ for all three antigens. Reciprocal GMP titers against H1N1pdm09 dropped from 201.6 to 50.4 , but formally remained on protective level $(>40)$. In sera taken on day 55 reciprocal GMP titers dropped to $40 / 40 / 25.2$ against H1N1pdm09/H3N2/B antigens. Reciprocal antibody titers in sera taken from animals of control group 3 (intranasal antigen only) on days $14,21,28,55$ were a.i. $<10$.

Data obtained in ELISA is presented in Figure 2. Antibody titers in sera of the animals from Group 1 taken on day 14 were 4064/1613/5120 against H1N1pdm09/H3N2/B antigens and remained statistically indistinguishable $(\mathrm{P}=0.95)$ to coinciding titers in sera taken on 21,28 and 55 day a.i. In experimental group 2 sera taken on day 14 showed titers to all antigens on the lowest limits of detection 100.8/40/40 (H1N1pdm09/H3N2/B). Second nasal injection of immunizing substance boosted reciprocal GMT ELISA titers to 806/3225/2031 (H1N1pdm09/H3N2/B) on day 21 (Figure 2B) and they were statistically indistinguishable $(\mathrm{P}=0.95)$ with corresponding titers in sera of the animals from Group 1. In sera taken on day 55 we detected decreasing of antibody titers against all antigens; H1N1pdm09 and $\mathrm{B}$ component remained statistically indistinguishable $(\mathrm{P}=0.95)$ with corresponding titers in sera of the animals from Group 1 and H3N2 
A

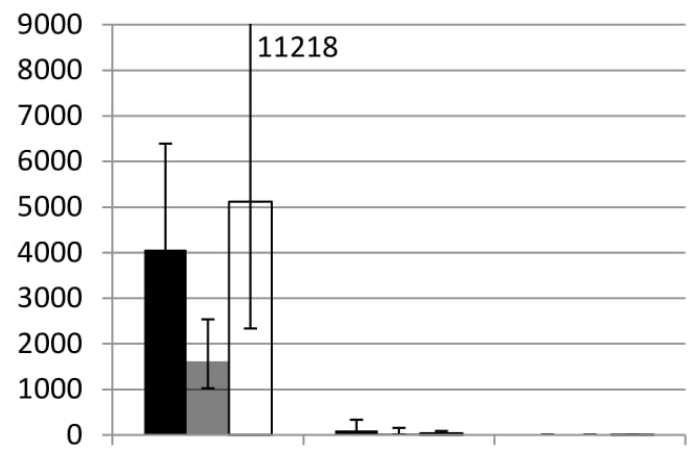

I.m. TIA 7,5 I.n. PTE 7,5 I.n. TIA 7,5 only
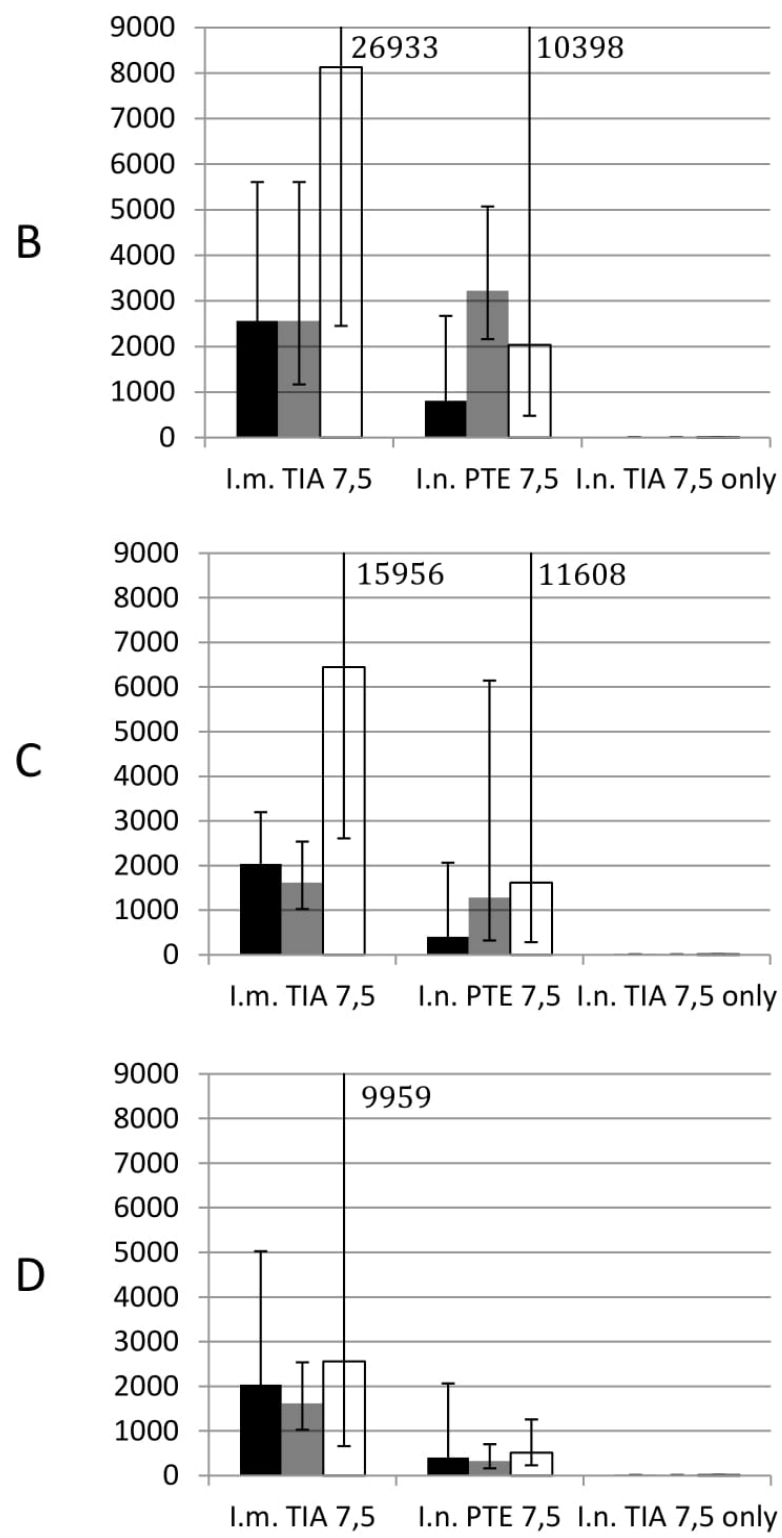

- A/California/7/2009 (H1N1)pdm09-like - A/Switzerland/9715293/2013 (H3N2)-like $\square$ B/Phuket/3073/2013-like

A/California/7/2009 (H1N1)pdm09-like A/Switzerland/9715293/2013 (H3N2)-like $\square$ B/Phuket/3073/2013-like

A/California/7/2009 (H1N1)pdm09-like A/Switzerland/9715293/2013 (H3N2)-like $\square$ B/Phuket/3073/2013-like

\section{- A/California/7/2009 (H1N1)pdm09-like A/Switzerland/9715293/2013 (H3N2)-like $\square$ B/Phuket/3073/2013-like}

Figure 2. Anti influenza virus titers in guinea pig sera measured in ELISA.

Reaction was performed with egg-derived antigens. Data expressed as reciprocal titers (C.I. P=0.95). I.m. TIA 7.5 - intramuscular triple influenza antigen $7.5 \mu$ g each (positive antigen control). I.n. PTE + TIA 7.5 - intranasal Polemonium caeruleum tuber extract + triple influenza antigen $7.5 \mu$ g each (experimental group). I.n. TIA 7.5 only - intranasal triple influenza antigen $7.5 \mu \mathrm{g}$ each (negative antigen control). A, B, C, D - 14, 21, 28, 55 days after immunization 
component dropped significantly $(\mathrm{P}=0.95)$ lower in comparison with corresponding titers in sera of the animals from Group 1. Antibody titers in sera taken from animals of control group 3 (intranasal antigen only) on days $14,21,28,55$ a.i. were below 2 -x background level signal against all used antigens.

Data obtained in ELISA supports main conclusions of HI analysis. Single intranasal injection did not cause immune response which can be compared with single intramuscular immunization, but sera titers are detectable in EILSA. After second intranasal injection boosting immunogenic effect was detected. Since day 21 a.i. today 55 titers detected in ELISA in the sera of animals from group 2 dually immunized intranasally were statistically indistinguishable $(\mathrm{P}=0.95)$ from corresponding titers from group 1 immunized intramuscularly, excluding titer against $\mathrm{H} 3$ antigen in sera taken on day 55.

\section{Discussion}

Adjuvant activities of saponins from medicinal herbs were widely described as well as a potential mechanism of action [17,18]. Direct antiviral activities of triterpene saponin isolated from Angallis arvensis, Primulaceae was shown against herpes simplex type 1, adenovirus type 6, vaccinia, vesicular stomatitis and poliovirus [19]. Thus, for vaccine development it is crucial to comply with pharmacopeia methods of effectiveness control.

Performed study shows that saponins from pharmacopeia plant Polemonium caeruleum has immunogenic activities in guinea pig model showed by pharmacopeia vaccine control method as hemagglutinin inhibition reaction. Saponins extracted from different plants (Polemonium caeruleum, Cyclamen europaeum) standardized by haemolitical reaction have almost the same immunogenic effects after intranasal administration performed identically [13]. Effectiveness of proposed immunization scheme, supposing dual intranasal injection on day 1 and 14, effective immunogenic saponin dose, was confirmed. The same time, these extracts have complicated chemical structure and concomitant plant substances. Individual specific features such are solubility, stability, dose dependant physiological action must be investigated beforehand. Overall, presented research shows that Polemonium caeruleum saponins can be studied as a candidate mucosal adjuvant for intranasal or oral viral or bacterial inactivated vaccines against respiratory pathogens and also investigated as immunotherapeutic agents against herpes simplex virus type 1 , type 2 and other viral and bacterial pathogens affecting mucous membranes.

\section{Acknowledgment}

Authors would like to appreciate State Research Center of Virology and Biotechnology Vector (SRC VB "Vector") Rospotrebnadzor Core Animal facilities for technical assistance.

\section{Compliance}

All institutional and national guidelines for the care and use of laboratory animals were followed. This article does not contain any studies with human participants performed by any of the authors.
This research study was funded by Russian Federal Service for Surveillance on Consumer Rights Protection and Human Wellbeing (Rospotrebnadzor). Authors declare that they have no conflicts of interest.

\section{References}

1. Forum of international respiratory societies (2017) The global impact of respiratory disease - Second edition. Sheffield, European Respiratory Society.

2. Darvishian M, Dijkstra F, van Doorn E (2017) Influenza vaccine effectiveness in the netherlands from 2003/2004 through 2013/2014: The importance of circulating influenza virus types and subtypes. PLoS One 12: e0169528. [Crossref]

3. Uhart M, Bricout H, Clay E, Largeron N (2016) Public health and economic impact of seasonal influenza vaccination with quadrivalent influenza vaccines compared to trivalent influenza vaccines in Europe. Human Vaccines \& Immunotherapeutics 12: 2259-2268. [Crossref]

4. Glueck R (2001) Pre-clinical and clinical investigation of the safety of a novel adjuvant for intranasal immunization. Vaccine 20: 42-44.

5. Glück U, Gebbers JO, Glück R (1999) Phase 1 evaluation of intranasal virosomal influenza vaccine with and without escherichia coli heat-labile toxin in adult volunteers. Journal of Virology 73: 7780-7786.

6. Mutsch M, Zhou W, Rhodes P (2004) Use of the inactivated intranasal influenza vaccine and the risk of bell's palsy in Switzerland. N Engl J Med 350: 896-903. [Crossref]

7. Kraan H, Soema P, Amorij JP, Kersten G (2017) Intranasal and sublingual delivery of inactivated polio vaccine. Vaccine $35: 2647-2653$

8. Ren ST, Zhang XM, Sun PF (2017) Intranasal immunization using mannatide as a novel adjuvant for an inactivated influenza vaccine and its adjuvant effect compared with MF59. PLoS ONE 12: e0169501. [Crossref]

9. Berezin VE, Bogoyavlenskiy AP, Turmagambetova AS (2013) Nanoparticles from Plant Saponins as Delivery System for Mucosal Influenza Vaccine. American Journal of Infectious Diseases and Microbiology 1: 1-4.

10. den Brok MH, Büll C, Wassink M, et al. (2016) Saponin-based adjuvants induce crosspresentation in dendritic cells by intracellular lipid body formation. Nat Commun 7: 13324. [Crossref]

11. Augustin JM, Kuzina V, Andersen SB, Bak S (2011) Molecular activities, biosynthesis and evolution of triterpenoid saponins. Phytochemistry 72: 435-457. [Crossref]

12. Pfaar O, Mullol J, Anders C, Hörmann K, Klimek L (2012) Cyclamen europaeum nasal spray, a novel phytotherapeutic product for the management of acute rhinosinusitis: a randomized double-blind, placebo-controlled trial. Rhinology 50: 37-44.

13. Evseenko VA, Kolosova NP, Gudymo AS (2019) Intranasal immunization of guinea pig with trivalent influenza antigen adjuvanted by Cyclamen europaeum tubers extract. Arch Virol 164: 243-247. [Crossref]

14. Laska G, Sieniawska E, Swiatek L, Zjawiony J, Khan S, et al. (2019) Phytochemistry and biological activities of Polemonium caeruleum L. Phytochemistry 30: 314-323.

15. Russian Federation pharmacopoeia 14 (2018), Vol. 4, pp. 6421-6428.

16. World Health Organization (2004) WHO manual on animal influenza diagnosis and surveillance.

17. Song X, Hu S (2009) Adjuvant activities of saponins from traditional Chinese medicinal herbs. Vaccine 27: 4883-4890. [Crossref]

18. Simões CMO, Amoros M, Girre L (1999) Mechanism of antiviral activity of triterpenoid saponins. Phytother Res 13: 323-328. [Crossref]

19. Amoros M, Fauconnier B, Girre RL (1987) In vitro antiviral activity of a saponin from Anagallis arvensis, Primulaceae, against herpes simplex virus and poliovirus. Antiviral Research 8: 13-25. [Crossref]

Copyright: $\odot 2020$ Evseenko VA. This is an open-access article distributed under the terms of the Creative Commons Attribution License, which permits unrestricted use, distribution, and reproduction in any medium, provided the original author and source are credited. 